\title{
Review of Charmonium and Bottomonium Quark State Production via Relativistic Heavy Ion Collisions
}

\author{
Leonard S. Kisslinger $D$ \\ Department of Physics, Carnegie Mellon University, Pittsburgh, PA 15213, USA; kissling@andrew.cmu.edu \\ Received: 4 December 2019; Accepted: 26 December 2019; Published: 10 January 2020 \\ check for \\ updates
}

\begin{abstract}
This is a review of the production of heavy quark states via relativistic heavy ion collisions in RHIC. The heavy quarks here are $c$, charm quark, and $b$, bottom quark. The states are charmonium meson states $\Psi(n S)$, with $\mathrm{n}=1,2$ and upsilon meson states $\mathrm{Y}(m S)$, with $\mathrm{m}=1,2,3$. Quantum Chromodynamics (QCD) sum rules were used to derive the result that the $\Psi(2 S)$ and $Y(3 S)$ are mixed hybrid states, which increase their production cross sections. We also review the $\Psi(n S)$ and $\mathrm{Y}(m S)$ production cross sections via $\mathrm{Cu}-\mathrm{Cu}$ and $\mathrm{Au}-\mathrm{Au}$ collisions, which are very important for this review of the production of heavy quark states in RHIC. The possible detection of the Quark Gluon Plasma (QGP) is also reviewed.
\end{abstract}

Keywords: Quantum Chromodynamics; heavy quark mixed hybrid mesons; relativistic heavy ion collisions

\section{Introduction}

First we review Quantum Chromodynamics (QCD).

The elementary fermions, with quantum spin $1 / 2$, are quarks. There are three generations of quarks $(\mathrm{u}, \mathrm{d}),(\mathrm{c}, \mathrm{s}),(\mathrm{t}, \mathrm{b})$. The $\mathrm{u}(\mathrm{up}), \mathrm{d}($ down), and $\mathrm{s}($ strange) quarks have small masses and are not needed in this review. The $\mathrm{t}(\mathrm{top})$ quark has a very large mass, with $m_{t} \simeq 173 \mathrm{GeV}$, while $m_{b} \simeq 4.18 \mathrm{GeV}[1]$.

The elementary boson, the gluon (g), has quantum spin 1, like the photon. The photon has an electromagnetic interaction $\mathrm{U}(1)$ with quarks while the gluon has a strong interaction $\mathrm{SU}(3)$ with quarks. Since the strong interaction is approximately 100 times larger than the electromagnetic interaction, perturbation theory does not work. Therefore Feynman diagrams used for Quantum Electrodynamics cannot be used for Quantum Chromodynamics as the higher order diagrams are larger than the lower order diagrams [2].

One nonperturbative QCD method involves Lattice Gauge Theory. The article by Andreas S. Kronfelld [3] gives a detailed description of how Lattice Gauge Theory can calculate QCD interactions using computers.

Another nonperturbative theory, which is used in our review of mixed hybrid heavy quark mesons, is the method of QCD sum rules. This method, developed by M.A. Shifman, A.I. Vainstein, and V.I. Zakharov [4], does not require large computers.

Standard mesons consist of a quark and antiquark: $\mid q \bar{q}>$. A top quark meson $\mid t \bar{t}>$ is so massive that none have been detected. The charmonium mesons are $\Psi(n S)$, with $n=1,2$. The upsilon mesons are $Y(m S)$, with $m=1,2,3$. It was the method of QCD sum rules that showed that the $\Psi(2 S)$ charmonium quark meson and the $Y(3 S)$ bottomonium quark mesons are mixed hybrid states, while all the other $\Psi(n S)$ and $Y(m S)$ are standard charmonium and bottomonium meson states. QCD, charmonium, bottomonium meson states were reviewed by N. Brambilla et.al. [5] and recent theoretical progress by A. Rothkopf [6]. 
The production of $\Psi(n S)$ and $\mathrm{Y}(m S)$ cross sections via $\mathrm{Cu}-\mathrm{Cu}$ and $\mathrm{Au}-\mathrm{Au}$ collisions is an extension of the theory [7] for p-p to $\mathrm{Cu}-\mathrm{Cu}$ and Au-Au collisions. In Ref [7]the color octet model [7-10] was used.

In Section 4, it is reviewed that experiments confirmed that the $\Psi(2 S)$ and $Y(3 S)$ are mixed normal and hybrid states by the ratios of $\Psi(2 S)$ to $J / \Psi(1 S)$ and $Y(3 S)$ to $Y(1 S)$ cross sections.

In Section 5, heavy quark state production in the color octet model via A-A collisions is reviewed, with the gluon distribution function $f_{g}$ needed for RHIC. The heavy quarks are $c$, charm quark, and $b$, bottom quark. The states are charmonium states $\Psi(n S)$, with $\mathrm{n}=1,2$ and upsilon states $\mathrm{Y}(m S)$, with $\mathrm{m}=1,2,3$. The $\Psi(2 S)$ and $\mathrm{Y}(3 S)$ are mixed hybrid states, which increased their production cross sections. We also review the $\Psi(n S)$ and $\mathrm{Y}(m S)$ production cross sections via $\mathrm{Cu}-\mathrm{Cu}$ and $\mathrm{Au}-\mathrm{Au}$ collisions, which is very important for this review of the production of heavy quark states via RHIC.

In Section 6 Cosmological Phase Transitions and the possible detection of the Quark Gluon Plasma (QGP) via RHIC by the production of heavy quark hybrid states are reviewed.

\section{Quantum Chromodynamics (QCD)}

In Quantum Chromodynamics (QCD) the elementary particles are quarks (Fermions) and gluons (Bosons).

Strong interactions are produced by quarks exchanging gluons, as illustrated in the figure below.

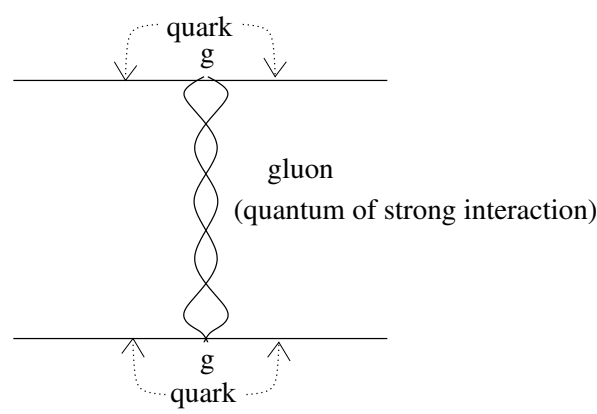

QCD (Quantum Chromodynamics): quark force via gluon exchange

STRONG FORCE

$$
\mathrm{g}^{2} \sim 1 \sim 100 \mathrm{x} \mathrm{e}^{2}
$$

Nonperturbative. Diagrams do not converge- single diagram no good

Color: Quarks have three colors. Color is due to the strong interaction, as electric charge is due to the electromagnetic interaction. A quark and antiquark can form a gluon, which has color.

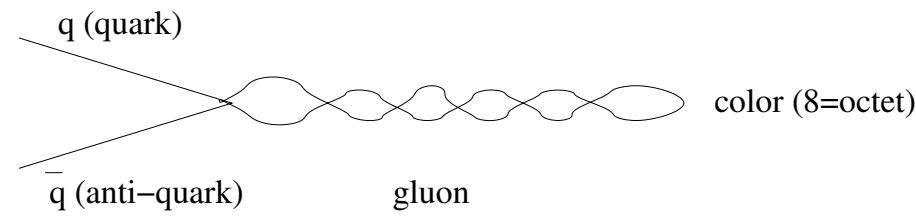

Note that particles with color, like gluons and quarks, cannot move freely in space. Particles which can move freely are baryons, like the proton and neutron, and mesons, which have no total color. The heavy quarks in this review are charm $c$ and bottom $b$.

Light mesons, such as the $\pi$, consist of light quarks $u, d$ and antiquarks $\bar{u}, \bar{d}$. We do not need them in this Review.

\section{Review of Mixed Hybrid Heavy Quark Mesons and the Color Octet Model}

In this section the hybrid heavy quark mesons and the color octet models are reviewed. 
The Method of QCD Sum Rules and Mixed Hybrid Heavy Quark Mesons

The starting point of the method of QCD sum rules [4] for finding the mass of a state A is the correlator,

$$
\Pi^{A}(x)=\left\langle\left|T\left[J_{A}(x) J_{A}(0)\right]\right|\right\rangle
$$

with |\rangle the vacuum state and the current $J_{A}(x)$ creating the states with quantum numbers A:

$$
J_{A}(x)|\rangle=c_{A}|A\rangle+\sum_{n} c_{n}|n ; A\rangle
$$

where $|A\rangle$ is the lowest energy state with quantum numbers $\mathrm{A}$, and the states $|n ; A\rangle$ are higher energy states.

The QCD sum rule is obtained by evaluating $\Pi^{A}$ in two ways. First, after a Fourier transform to momentum space, a dispersion relation gives the left-hand side (lhs) of the sum rule:

$$
\Pi(q)_{\mathrm{lhs}}^{A}=\frac{\operatorname{Im} \Pi^{A}\left(M_{A}\right)}{\pi\left(M_{A}^{2}-q^{2}\right)}+\int_{s_{o}}^{\infty} d s \frac{\operatorname{Im} \Pi^{A}(s)}{\pi\left(s-q^{2}\right)}
$$

where $M_{A}$ is the mass of the state $A$ (assuming zero width) and $s_{0}$ is the start of the continuum.

Next $\Pi^{A}(q)$ is evaluated by an operator product expansion (O.P.E.), giving the right-hand side (rhs) of the sum rule

$$
\Pi(q)_{\mathrm{rhs}}^{A}=\sum_{k} c_{k}(q)\left\langle 0\left|\mathcal{O}_{k}\right| 0\right\rangle
$$

where $c_{k}(q)$ are the Wilson coefficients and $\left\langle 0\left|\mathcal{O}_{k}\right| 0\right\rangle$ are gauge invariant operators.

A Borel transform, $\mathcal{B}$, replaces the $q$ variable by the Borel mass, $M_{B}$,

$$
\mathcal{B}=\left.\lim _{q^{2}, n \rightarrow \infty} \frac{1}{(n-1) !}\left(q^{2}\right)^{n}\left(-\frac{d}{d q^{2}}\right)^{n}\right|_{q^{2} / n=M_{B}^{2}} .
$$

The final QCD sum rule has the form

$$
\mathcal{B} \Pi(q)_{\mathrm{lhs}}^{A} \quad=\quad \mathcal{B} \Pi(q)_{\mathrm{rhs}}^{A}
$$

Using the method of QCD sum rules it was shown [11] that the $\Psi(2 S)$ and $Y(3 S)$ are approximately 50-50 mixtures of standard quarkonium and hybrid quarkonium states:

$$
\begin{aligned}
& |\Psi(2 S)>=-0.7| c \bar{c}(2 S)>+\sqrt{1-0.5} \mid c \bar{c} g(2 S)> \\
& |\mathrm{Y}(3 S)>=-0.7| b \bar{b}(3 S)>+\sqrt{1-0.5} \mid b \bar{b} g(3 S)>,
\end{aligned}
$$

with a $10 \%$ uncertainty [12] in the QCD sum rule estimate of the mixing probabilitiy, while

$$
\begin{aligned}
\mid J / \Psi(1 S)> & =\mid c \bar{c}(1 S)> \\
\mid \mathrm{Y}(n S)> & =\mid b \bar{b}(n S)>
\end{aligned}
$$

for $n \neq 3$.

For the $\Psi(2 S)$ state, about a 50-50 meson-hybrid meson [11], the mass of the lowest-energy mixed charmonium-hybrid is shown in Figure 1. 


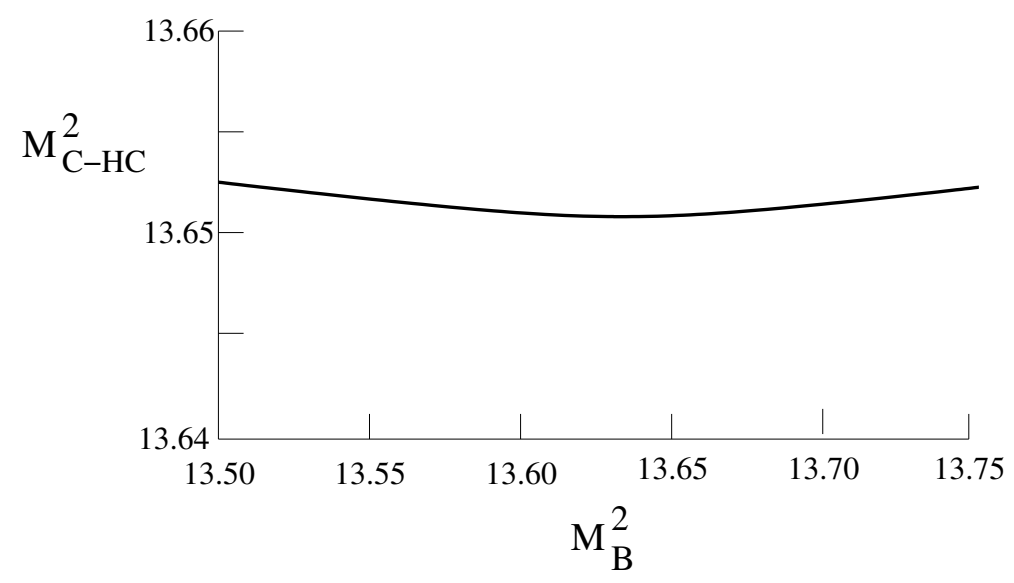

Figure 1. Mixed charmoniun-hybrid charmonium mass $=3.69 \mathrm{GeV}[11]$.

Therefore, in Ref [11] the mass of the lowest energy mixed charmonium-hybrid charmonium was found to be about the mass of the $\Psi(2 S)$ state, $3.69 \mathrm{GeV}$.

The cross sections for charmonium and bottomonium production in the color octet model, explained in Ref [13], are based on the cross sections obtained from the matrix elements for quark-antiquark and gluon-gluon octet fusion to a hadron $\mathrm{H}$, illustrated in Figure 2.

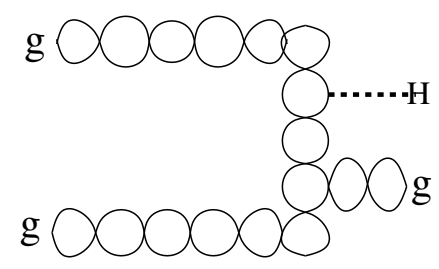

octet gluon fusion

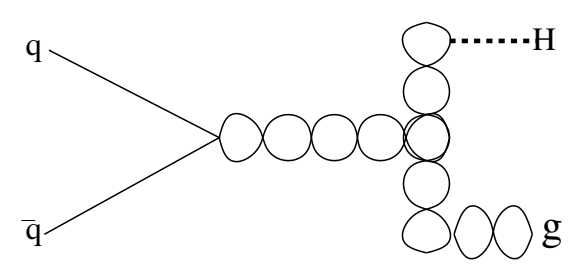

octet quark-antiquark fusion

Figure 2. Gluon and quark-antiquark color octet fusion producing hadron $\mathrm{H}$.

With $q \bar{q}$ models pp cross section ratios are [7] $\sigma(2 S) / \sigma(1 S) \simeq 0.039, \sigma(3 S) / \sigma(1 S) \simeq 0.0064$. On the other hand, for gluonic interactions with quarks there is an enhancement factor of $\pi^{2}$, for purely hybrid states, as illustrated in Figure 2. For states that are approximately $50 \%$ hybrid, this gives an enhancement factor of $\pi^{2} / 4$, with a $10 \%$ uncertainty, which accounts for the enhanced cross section ratios discussed above, in Ref [7], and in Figure 3.

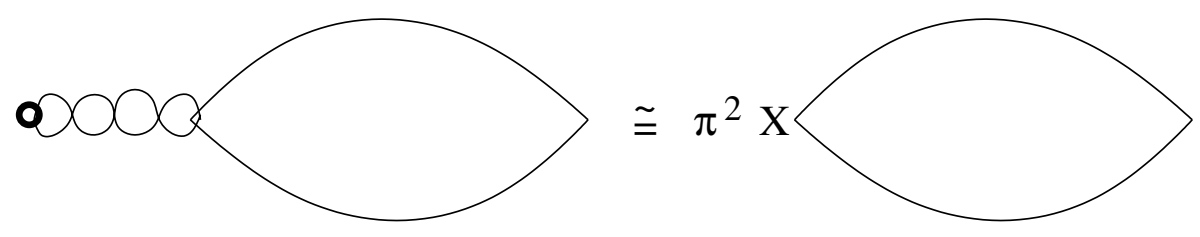

Figure 3. External field method for $\Psi(2 S)$ and $Y(3 S)$ states.

\section{QCD Sum Rules and Mixed Heavy Quark Hybrid Meson States}

The starting point of the method of QCD sum rules [4] is the correlator

$$
\Pi^{A}(x)=\left\langle\left|T\left[J_{A}(x) J_{A}(0)\right]\right|\right\rangle
$$


with |\rangle the vacuum state and the current $J_{A}(x)$ creates the states with quantum numbers A. For the charmonium states, $J_{A}=J_{c}$ is

$$
J_{c}=f J_{c \bar{c}}+\sqrt{1-f^{2}} J_{c \bar{g} g}
$$

where $J_{c \bar{c}}$ creates a normal charmonium state and $J_{c \bar{c} g}$ creates a hybrid state with an active gluon. As discussed in the previous subsection $f \simeq-\sqrt{2}$ for the $\Psi(2 S)$ and $Y(3 S)$ and $f \simeq 1.0$ for the other charmonium and bottomonium states [11].

Experimental Verification that the $\Psi(2 S)$ and $\mathrm{Y}(3 S)$ are Mixed Hybrid Heavy Quark Mesons

$\Psi$ and $\mathrm{Y}$ production $\mathrm{Cu}-\mathrm{Cu}$ Collisions for $\mathrm{E}=200 \mathrm{GeV}$ [14] are shown in Figures 4-7. The dashed curves are for the standard model.

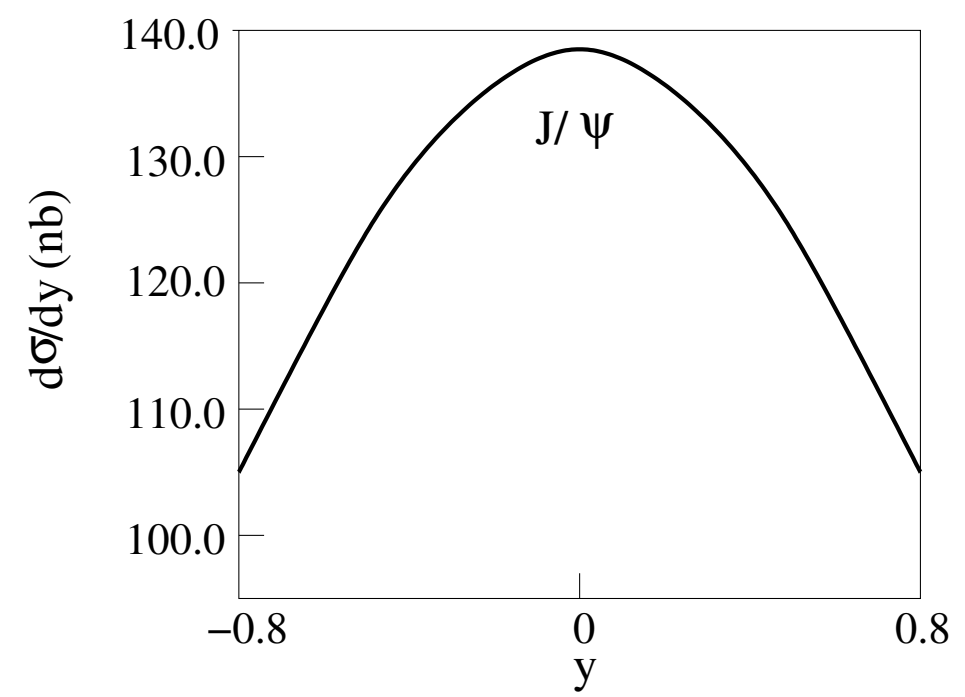

Figure 4. $\mathrm{d} \sigma / \mathrm{dy}$ for $\mathrm{E}=200 \mathrm{GeV} \mathrm{Cu}-\mathrm{Cu}$ collisions producing $J / \Psi$ [14].

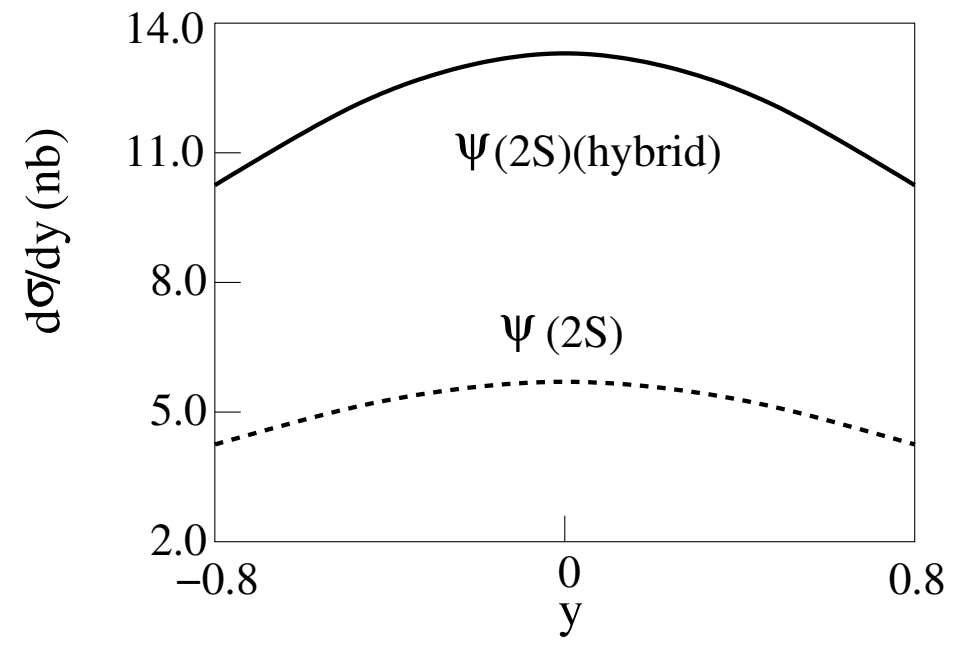

Figure 5. $\mathrm{d} \sigma / \mathrm{dy}$ for $\mathrm{E}=200 \mathrm{GeV} \mathrm{Cu}-\mathrm{Cu}$ collisions producing $\Psi(2 S)$ [14]. 


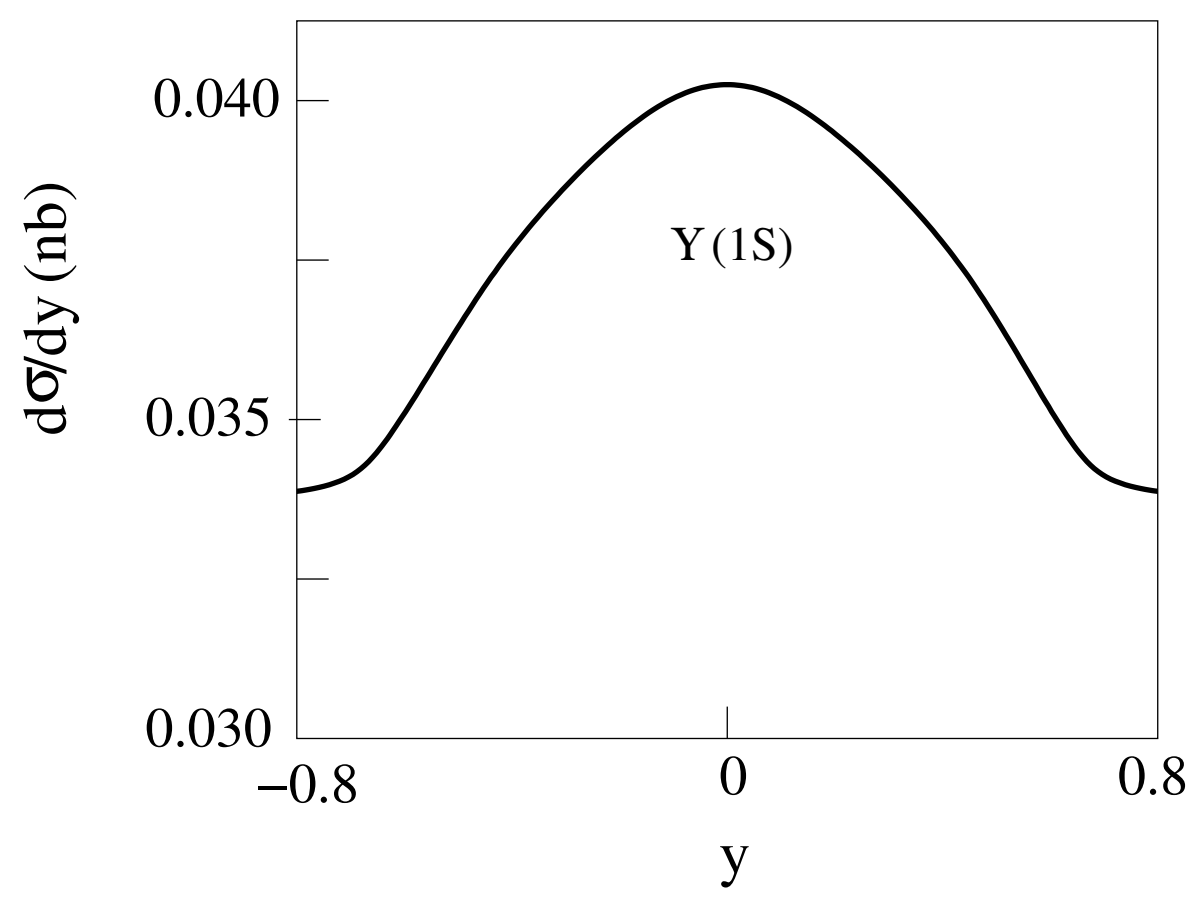

Figure 6. $\mathrm{d} \sigma / \mathrm{dy}$ for $\mathrm{E}=200 \mathrm{GeV} \mathrm{Cu}-\mathrm{Cu}$ collisions producing $\mathrm{Y}(1 S)$ [14].

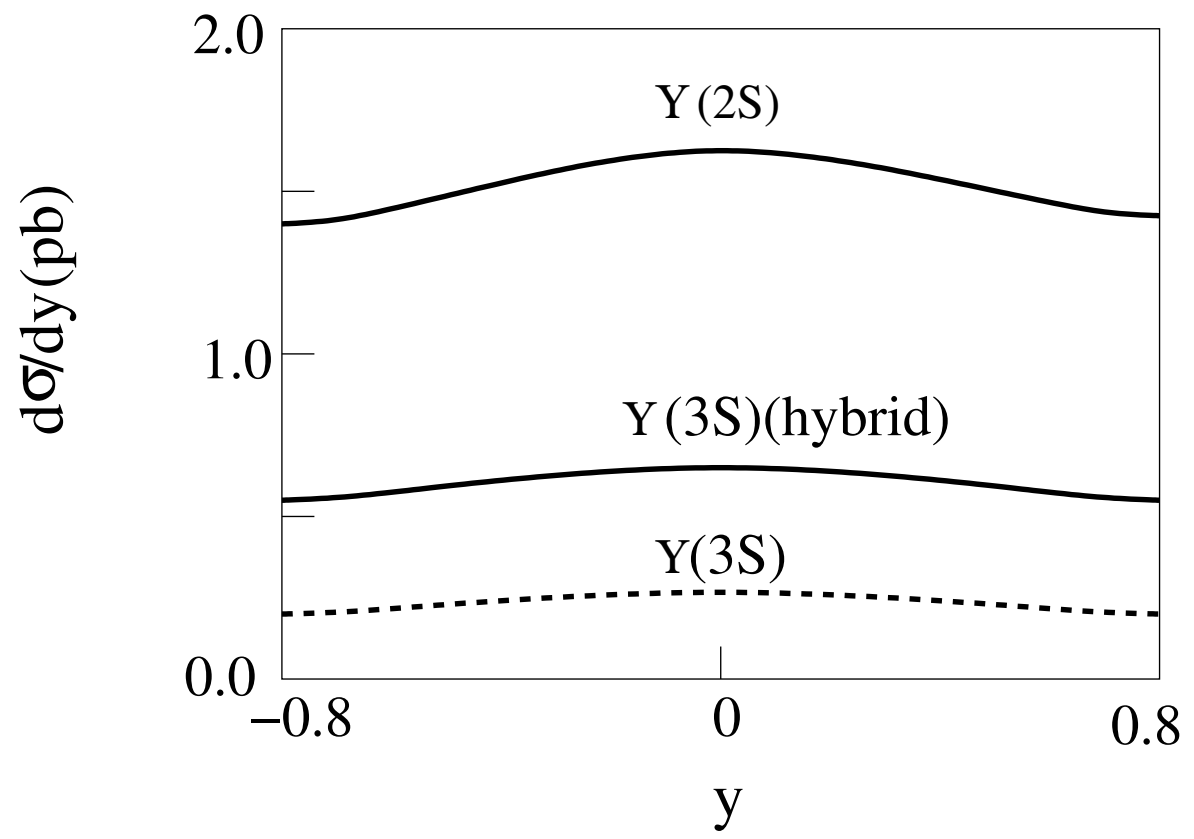

Figure 7. $\mathrm{d} \sigma / \mathrm{dy}$ for $\mathrm{Cu}-\mathrm{Cu}$ collisions producing $\mathrm{Y}(2 S), \mathrm{Y}(3 S)$ [14].

Tests of the mixed hybrid theory for $\Psi(2 S)$ and $Y(3 S)$ states using ratios of cross sections for $\mathrm{Cu}-\mathrm{Cu}$ Collisions at $\mathrm{E}=200 \mathrm{GeV}$ : Since the absolute magnitude of $\mathrm{d} \sigma /$ dy for production of $\Psi(2 S)$ states via $\mathrm{Cu}-\mathrm{Cu}$ collisions is not certain, due to uncertainty in the normalization of the states, the tests of the theory [14] were carried out using ratios of cross sections, which can be compared to experiments.

From Figures 1 and 2 the ratios of $\Psi(2 S)$ to $J / \Psi(1 S)$ for the standard model (st) and the mixed hybrid theory (hy) for A-A (including $\mathrm{Cu}-\mathrm{Cu}$ ) collisions are 


$$
\begin{aligned}
\sigma(\Psi(2 S)) /\left.\sigma(J / \Psi(1 S))\right|_{s t-A--A} & \simeq 0.27 \\
\sigma(\Psi(2 S)) /\left.\sigma(J / \Psi(1 S))\right|_{h y-A--A} & \simeq 0.52 \pm 0.05
\end{aligned}
$$

while the experimental result [15] is

$$
\sigma(\Psi(2 S)) / \sigma(J / \Psi(1 S) \simeq 0.59
$$

which shows that the mixed hybrid theory for the $\Psi(2 S)$ state is consistent with experiment, while the standard $\mid c \bar{c}(2 S)>$ is not.

From studies of heavy quark state production in p-p collisions theoretical results for the nature of $\mathrm{Y}(3 S)$ state found [7]

$$
\begin{aligned}
& \sigma(\mathrm{Y}(3 S)) / \sigma(\mathrm{Y}(1 S)) \simeq 0.04 \text { standard } \\
& \sigma(\mathrm{Y}(3 S)) / \sigma(\mathrm{Y}(1 S)) \simeq 0.147-0.22 \text { hybrid }
\end{aligned}
$$

compared to the experimental result [16]

$$
\sigma(\mathrm{Y}(3 S)) / \sigma(\mathrm{Y}(1 S)) \simeq 0.12-0.16
$$

Therefore, the $Y(3 S)$ state as well as the $\Psi(2 S)$ state has been shown to be a heavy quark mixed hybrid state.

\section{5. $J / \Psi, \Psi(2 S)$ and $\mathrm{Y}(n S)$ Production in $\mathrm{Cu}-\mathrm{Cu}$ and Au-Au Collisions with $\sqrt{s_{p p}}=200 \mathrm{GeV}$}

The differential rapidity cross section for the production of a heavy quark state with in the color octet model in A-A collisions is given by

$$
\frac{d \sigma_{A A \rightarrow \Phi}}{d y}=R_{A A}^{E} N_{b i n}^{A A}<\frac{d \sigma_{p p} \rightarrow \Phi}{d y}>
$$

where $R_{A A}^{E}$ is the product of the nuclear modification factor $R_{A A}$ and $S_{\Phi}$, the dissociation factor after the state $\Phi$ (a charmonium or bottomonium state) is formed. $N_{b i n}^{A A}$ is the number of binary collisions in the A-A collision, and $<\frac{d \sigma_{p p \rightarrow \Phi(\lambda=0)}}{d y}>$ is the differential rapidity cross section for $\Phi$ production via nucleon-nucleon collisions in the nuclear medium.

Experimental studies show that for $\sqrt{s_{p p}}=200 \mathrm{GeV} R_{A A}^{E} \simeq 0.5$ both for $\mathrm{Cu}-\mathrm{Cu}$ [17] and $\mathrm{Au}-\mathrm{Au}$ [18]. The number of binary collisions are $N_{b i n}^{A A}=51.5$ for $\mathrm{Cu}-\mathrm{Cu}$ [19] and 258 for $\mathrm{Au}-\mathrm{Au}$. The differential rapidity cross section for pp collisions for $\sqrt{s_{p p}}=200 \mathrm{GeV}$ in terms of $f_{g}$ [7], the gluon distribution function is

$$
<\frac{d \sigma_{p p \rightarrow \Phi(\lambda=0)}}{d y}>\quad=\quad A_{\Phi} \frac{1}{x(y)} f_{g}(\bar{x}(y), 2 m) f_{g}(a / \bar{x}(y), 2 m) \frac{d x}{d y},
$$

with

$$
f_{g}(x) \simeq 275.14-6167.6 x+36871.3 x^{2},
$$

with

$$
x(y)=0.5\left[\frac{m}{s}(\exp y-\exp (-y))+\sqrt{\left(\frac{m}{s}(\exp y-\exp (-y))\right)^{2}+4 a}\right]
$$


and

$$
\begin{aligned}
\bar{x}(y) & =x(y)\left(1+\frac{\xi_{g}^{2}\left(A^{1 / 3}-1\right)}{Q^{2}}\right) \\
x(y) & =0.5\left[\frac{m}{\sqrt{s_{p p}}}(\exp y-\exp (-y))+\sqrt{\left(\frac{m}{\sqrt{s_{p p}}}(\exp y-\exp (-y))\right)^{2}+4 a}\right],
\end{aligned}
$$

with [19] $\xi_{g}^{2}=.12 \mathrm{GeV}^{2}$. For $J / \Psi Q^{2}=10 \mathrm{GeV}^{2}$, so $\bar{x}=1.058 x$ for Au and $\bar{x}=1.036 x$ for $\mathrm{Cu}$, while for $Y(1 S) Q^{2}=100 \mathrm{GeV}^{2}$, so $\bar{x}=1.006 x$ for Au and $\bar{x}=1.004 x$ for $\mathrm{Cu}$.

From this we find the differential rapidity cross sections as shown in the following figures for $J / \Psi, \Psi(2 S)$ and $\mathrm{Y}(1 S), \mathrm{Y}(2 S), \mathrm{Y}(3 S)$ production via $\mathrm{Cu}-\mathrm{Cu}$ and $\mathrm{Au}-\mathrm{Au}$ collisions at RHIC $(\mathrm{E}=200 \mathrm{GeV})$, with $\Psi(2 S), \mathrm{Y}(3 S)$ enhanced by $\pi^{2} / 4$ as discussed above. The absolute magnitudes are uncertain, and the shapes and relative magnitudes are our main prediction.

The differential cross sections $\mathrm{d} \sigma / \mathrm{dy}$ are shown for $\Psi(m S)$ and $\mathrm{Y}(1 n S)$ with $\mathrm{m}=1,2$ and $\mathrm{n}=1,2,3$ in Figures 8-15.

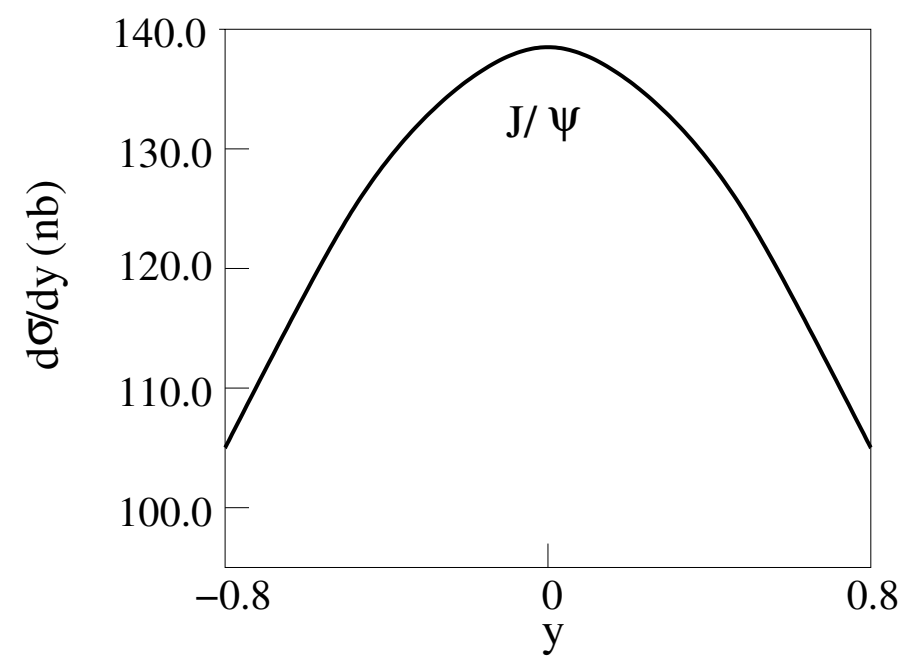

Figure 8. $\mathrm{d} \sigma /$ dy for $2 \mathrm{~m}=3 \mathrm{GeV}, \mathrm{E}=200 \mathrm{GeV} \mathrm{Cu}-\mathrm{Cu}$ collisions producing $J / \Psi$ with $\lambda=0$ [14].

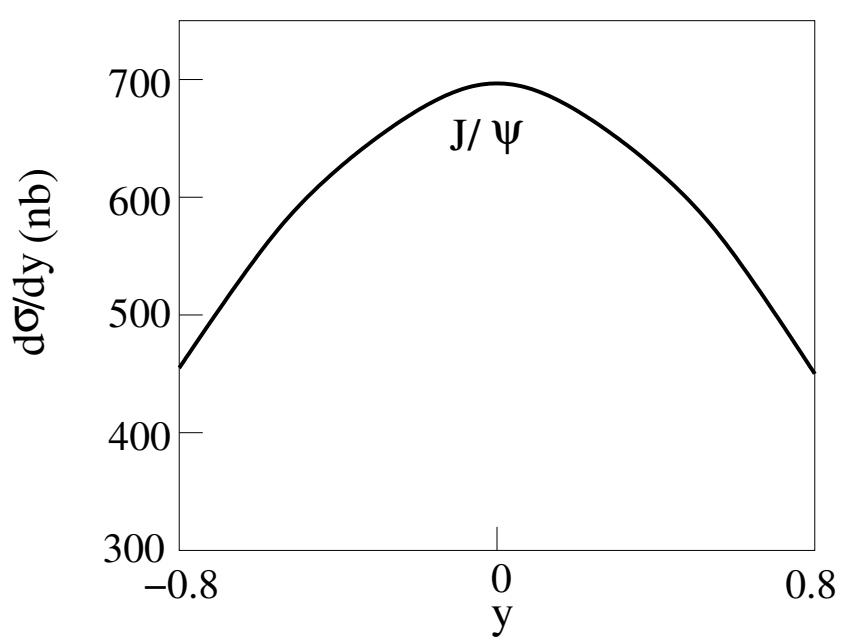

Figure 9. $\mathrm{d} \sigma / \mathrm{dy}$ for $2 \mathrm{~m}=3 \mathrm{GeV}, \mathrm{E}=200 \mathrm{GeV} \mathrm{Au}$-Au collisions producing $J / \Psi$ with $\lambda=0$ [14]. 


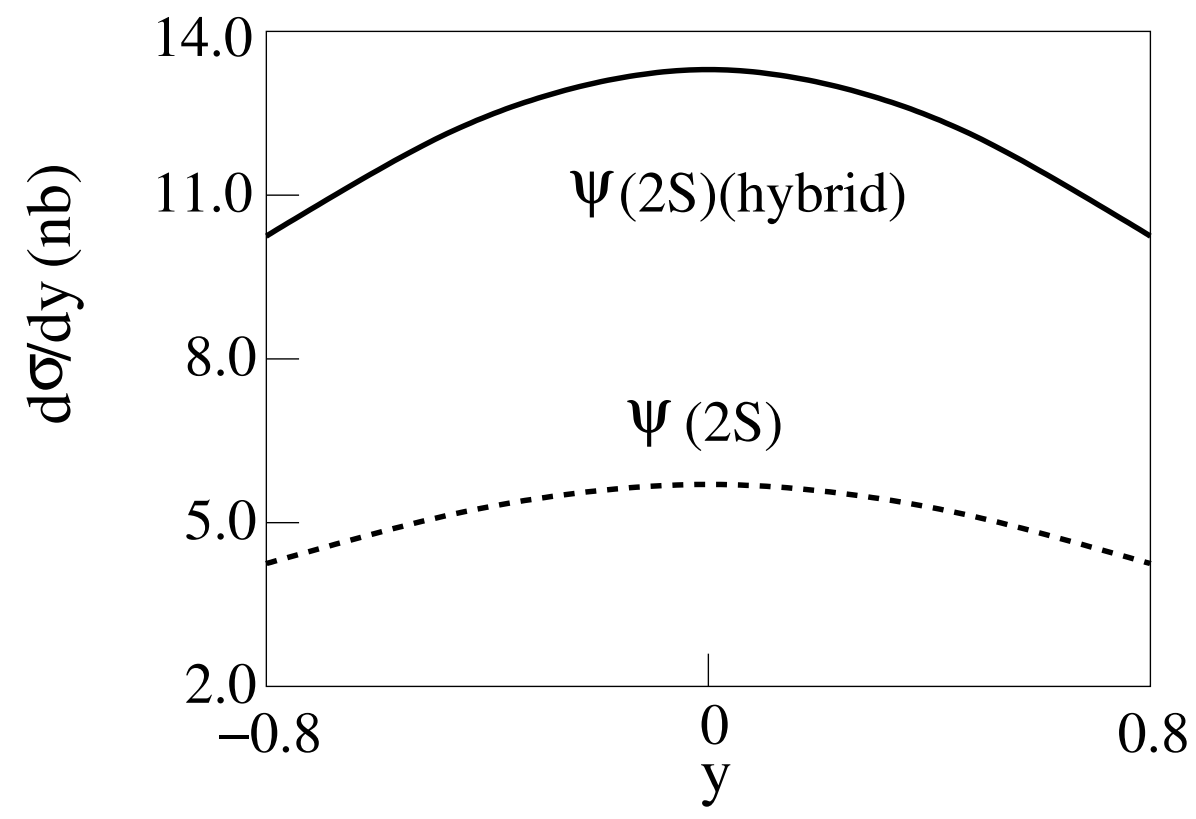

Figure 10. $\mathrm{d} \sigma / \mathrm{dy}$ for $2 \mathrm{~m}=3 \mathrm{GeV}, \mathrm{E}=200 \mathrm{GeV} \mathrm{Cu}-\mathrm{Cu}$ collisions producing $\Psi(2 S)$ with $\lambda=0$. The dashed curve is for the standard $c \bar{c}$ model [14].

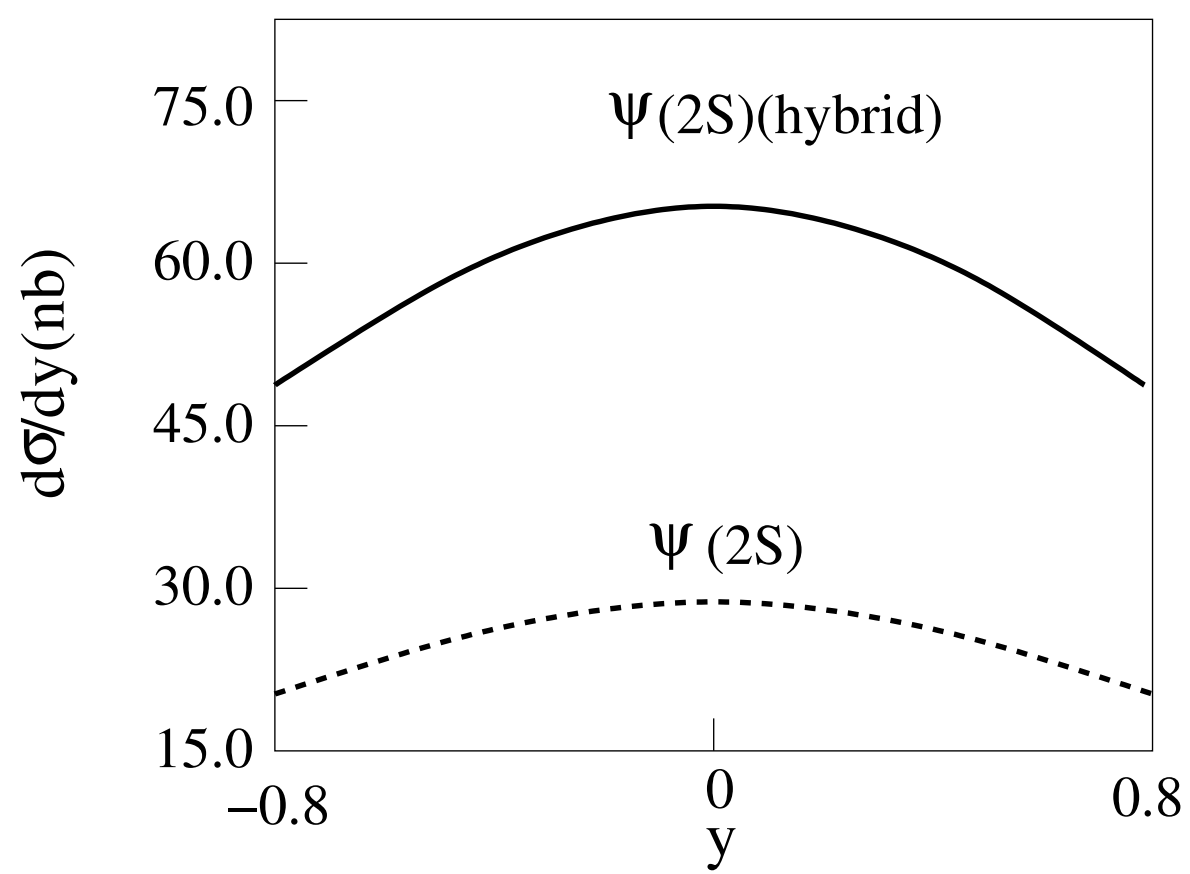

Figure 11. $\mathrm{d} \sigma / \mathrm{dy}$ for $2 \mathrm{~m}=3 \mathrm{GeV}, \mathrm{E}=200 \mathrm{GeV}$ Au-Au collisions producing $\Psi(2 S)$ with $\lambda=0$. The dashed curve is for the standard $c \bar{c}$ model [14]. 


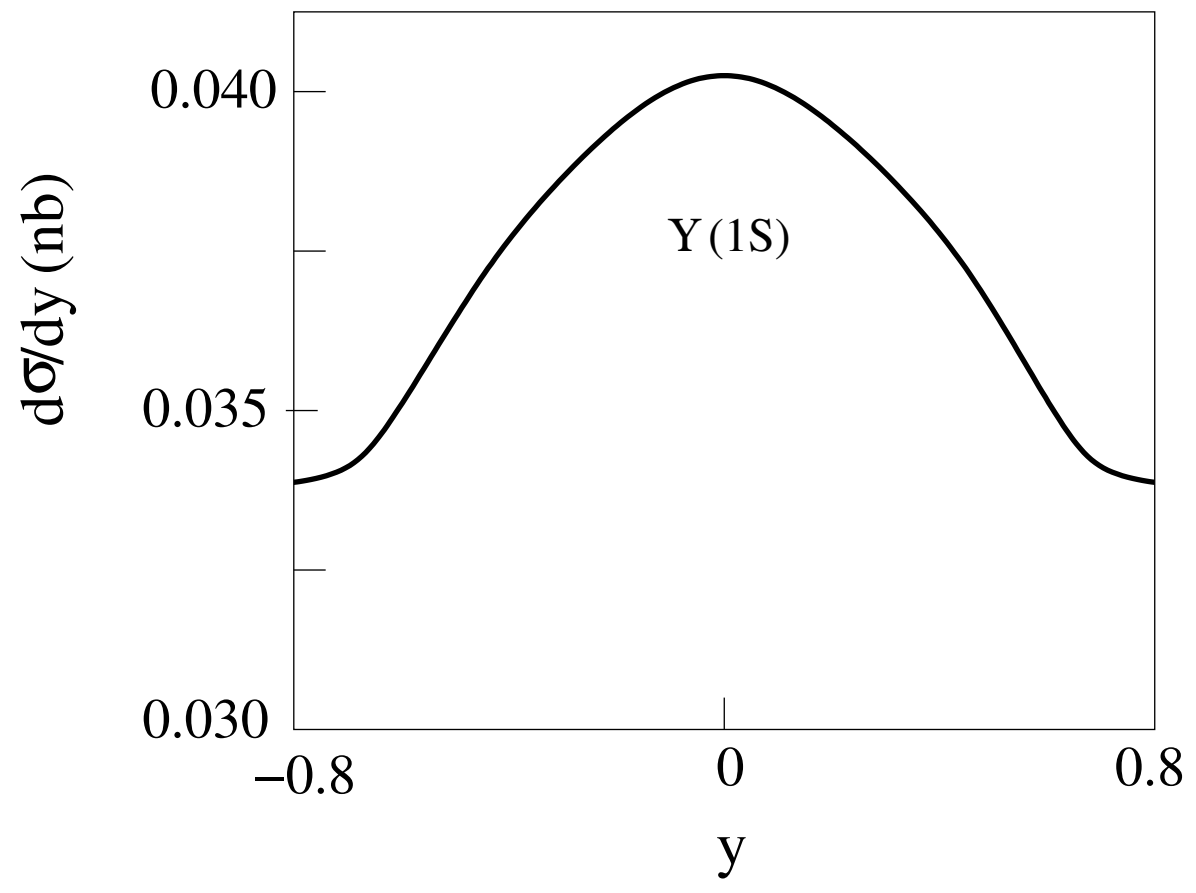

Figure 12. $\mathrm{d} \sigma / \mathrm{dy}$ for $2 \mathrm{~m}=10 \mathrm{GeV}, \mathrm{E}=200 \mathrm{GeV} \mathrm{Cu}-\mathrm{Cu}$ collisions producing $\mathrm{Y}(1 S)$ with $\lambda=0$ [14].

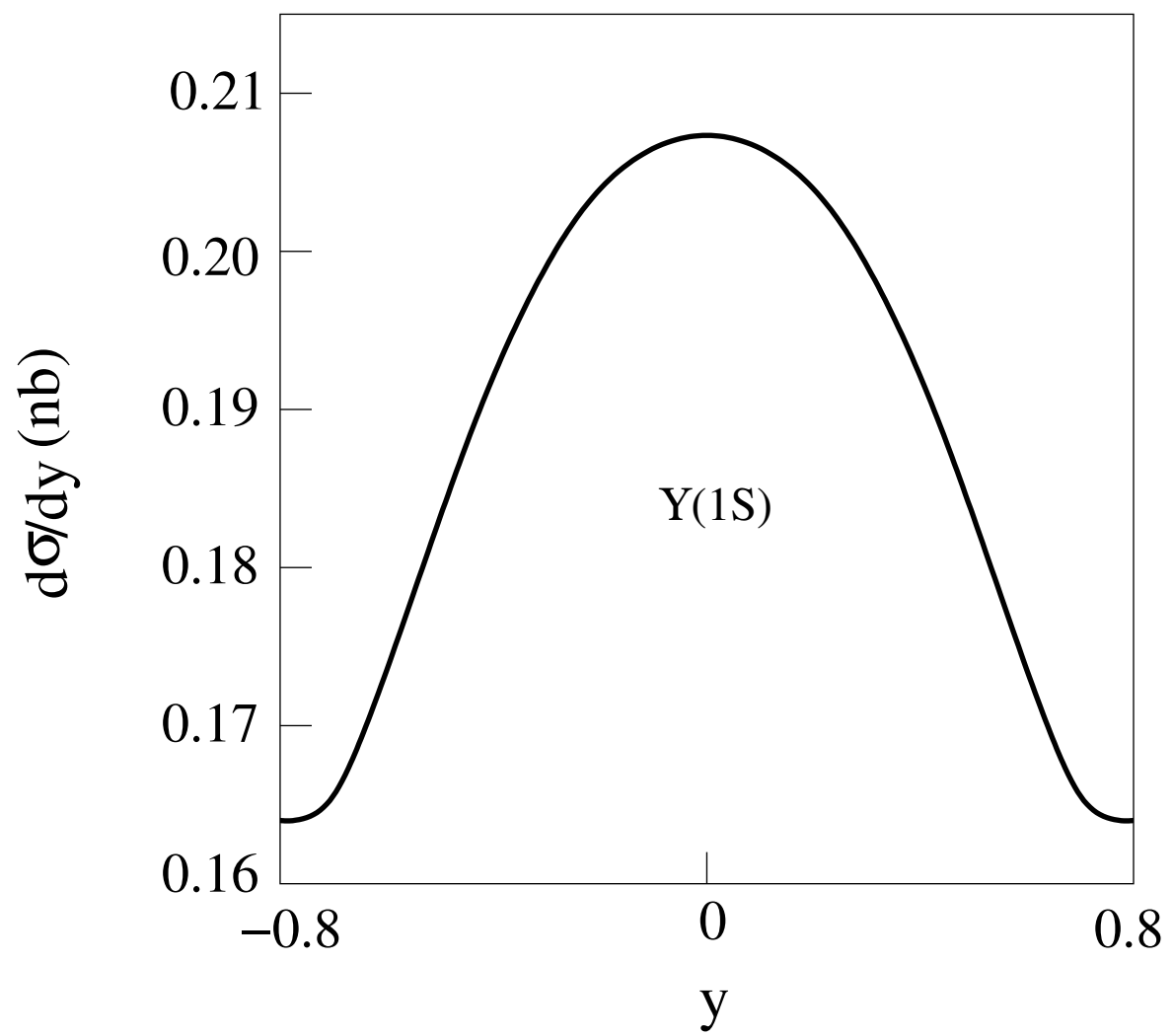

Figure 13. $\mathrm{d} \sigma / \mathrm{dy}$ for $2 \mathrm{~m}=10 \mathrm{GeV}, \mathrm{E}=200 \mathrm{GeV}$ Au-Au collisions producing $\mathrm{Y}(1 S)$ with $\lambda=0$ [14]. 


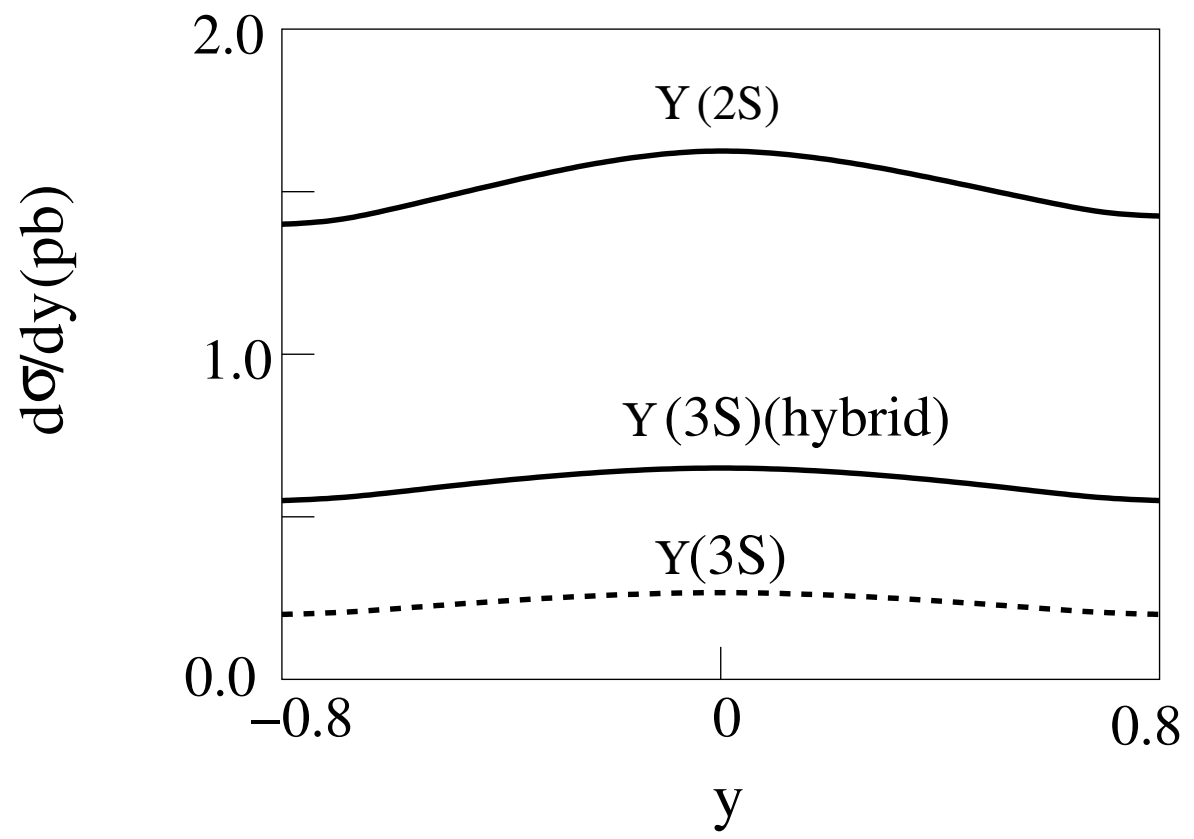

Figure 14. $\mathrm{d} \sigma / \mathrm{dy}$ for $2 \mathrm{~m}=10 \mathrm{GeV}, \mathrm{E}=200 \mathrm{GeV} \mathrm{Cu}-\mathrm{Cu}$ collisions producing $\mathrm{Y}(2 S), \mathrm{Y}(3 S)$ with $\lambda=0$. For $\mathrm{Y}(3 S)$ the dashed curve is for the standard $b \bar{b}$ model [14].

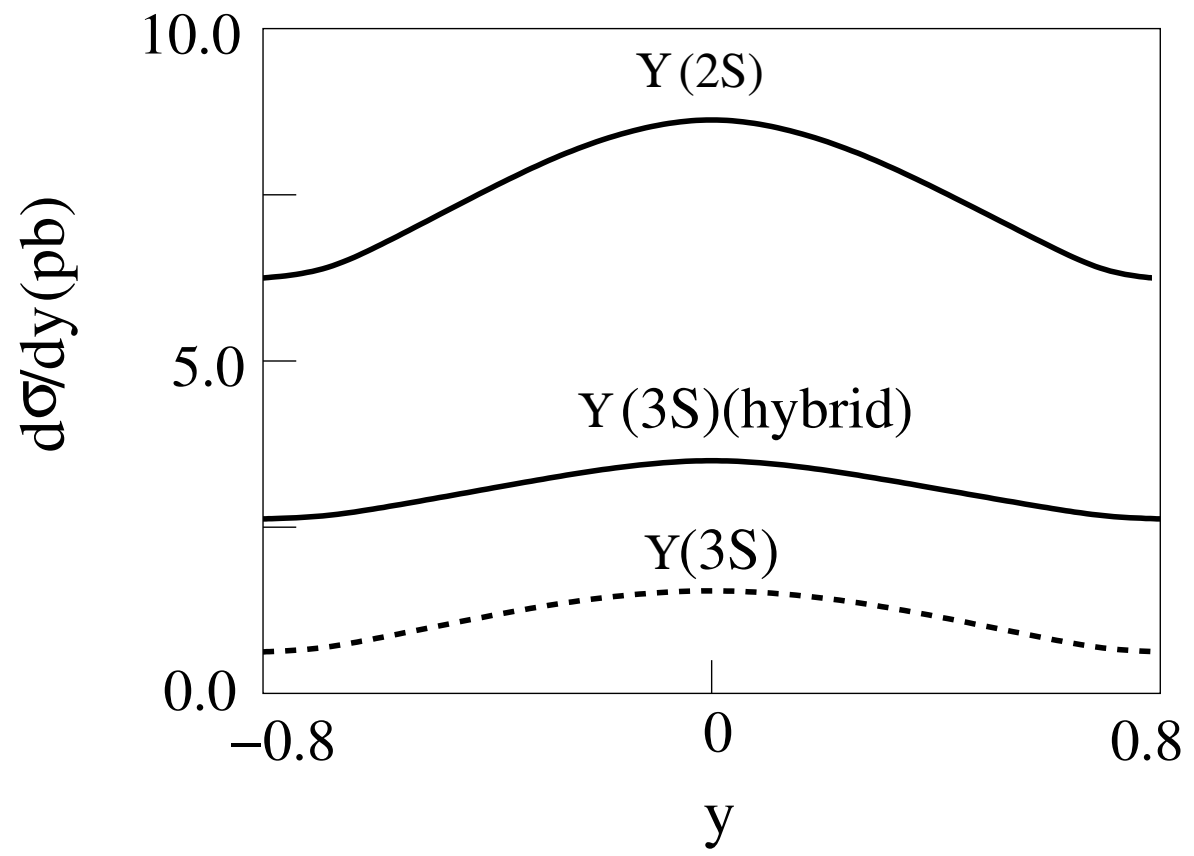

Figure 15. $\mathrm{d} \sigma / \mathrm{dy}$ for $2 \mathrm{~m}=10 \mathrm{GeV}, \mathrm{E}=200 \mathrm{GeV}$ Au-Au collisions producing $\mathrm{Y}(2 S), \mathrm{Y}(3 S)$ with $\lambda=0$. For $\mathrm{Y}(3 S)$ the dashed curve is for the standard $b \bar{b}$ model [14].

\section{Cosmological Phase Transitions}

Let us define $\mid 0, T>$ the state of the Universe with a temperature $T$ since there is thermal equilibrium with no flow of thermal energy.

If there is a Cosmological first order phase transition, then there is a critical temperature $T_{\mathcal{C}}$, and with the operator $A$ depending on the phase transition. 


$$
<0, T|A| 0, T>_{T<T_{\mathcal{C}}}-<0, T|A| 0, T>_{T>T_{\mathcal{C}}} \quad=\quad \Delta A
$$

$\Delta A=$ latent heat of the phase transition.

The Electroweak Phase Transition (EWPT) occured at the time $t \simeq 10^{-11} \mathrm{~s}$. The critical temperature of the EWPT is $T_{\mathcal{C}}(\mathrm{EWPT}) \simeq 125 \mathrm{GeV}$. For the EWPT the operator $A$ is $\phi$, the Higgs field, and

$$
\begin{aligned}
& <0, T|\phi| 0, T>_{T<T_{\mathcal{C}}}-<0, T|\phi| 0, T>_{T>T_{\mathcal{C}}}=\Delta \phi \\
= & \text { Higgs Mass } \simeq 125 \mathrm{GeV}=\text { the latent heat of the EWPT } .
\end{aligned}
$$

During the EWPT:

(1) all particles except the photon got their mass,

(2) magnetic fields were created,

(3) baryogenesis occured: more quarks than antiquarks.

The EWPT is reviewed in detail in Ref [20]. Next we discuss the QCDPT.

The QCD phase transition, QCDPT, occured at the time $t \simeq 10^{-5} \mathrm{~s}$, with the critical temperature for the QCDPT $T_{C}^{Q C D P T} \simeq 150 \mathrm{MeV}$. The Latent Heat for the QCD Phase Transition (QCDPT) is the Quark Condensate, which we now define.

$$
\begin{array}{rll}
q(x) & = & \text { quark field } \\
\bar{q}(x) & = & \text { antiquark field } \\
\mid 0, T> & = & \text { vacuum state temperature }=\mathrm{T} \\
<0, T|\bar{q}(x) q(x)| 0, T> & = & \text { quark condensate } \\
& = & \text { vacuum expectation value of } \overline{\mathrm{q}}(\mathrm{x}) \mathrm{q}(\mathrm{x})
\end{array}
$$

The quark condensate $<0, T|\bar{q}(x) q(x)| 0, T>$ changes during the QCDPT:

$$
\begin{aligned}
<0, T|\bar{q}(x) q(x)| 0, T> & =0 \text { in quark gluon plasma phase } \mathrm{T}>\mathrm{T}_{\mathrm{C}}^{\mathrm{QCDPT}} \\
& \simeq(.23 \mathrm{GeV})^{3} \text { in hadron phase } \mathrm{T}<\mathrm{T}_{\mathrm{C}}^{\mathrm{QCDPT}}
\end{aligned}
$$

Therefore

$$
\Delta<0, T|\bar{q}(x) q(x)| 0, T>\quad=\quad \text { latent heat of the QCDPT }=(.23 \mathrm{GeV})^{3}
$$

\subsection{Detection of the QGP via Hybrid Mesons Produced by RHIC}

As discussed above, during the evolution of the early universe there were two important Cosmological Phase Transitions: The Electorpeak Phase Transition when all particles except the photon got their mass, and the QCD phase transition, QCDPT, the transition from a universe with dense matter with quarks and gluons, called the quark gluon plasma (QGP), to our universe with protons and neutrons and other hadrons. The QCDPT is reviewed since hybrid mesons and RHIC are essential for the detection of the QGP.

The critical temperature $T_{\mathcal{C}}^{Q C D P T} \simeq 150 \mathrm{MeV}$. During the time that $T=T_{\mathcal{C}}^{Q C D P T}$ bubbles of our universe nucleated within the QGP, as shown in Figure 16. 


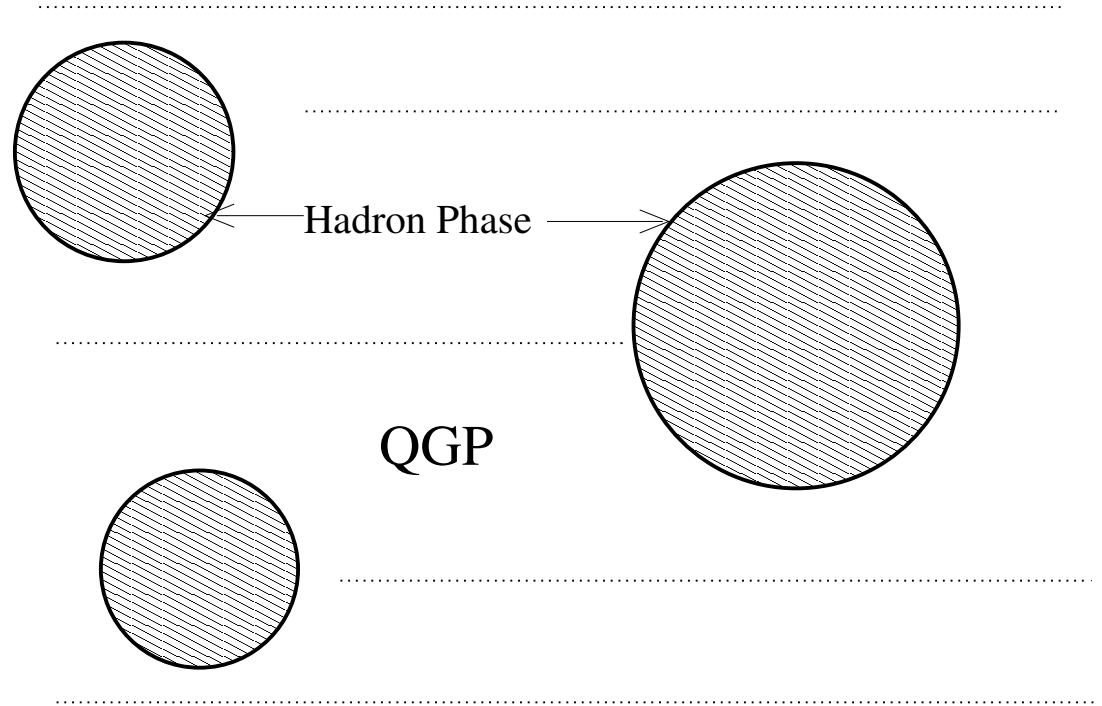

Figure 16. Hadron phase forming within the Quark Gluon Plasma (QGP) during the Quantum Chromodynamics Phase Transition (QCDPT).

\subsection{Creation and Detection of the QGP via A-A Collisions}

A main goal of the study of heavy quark state production in A-A colisions is the detection of the Quark Gluon Plasma (QGP).

The energy of the atomic nuclei must be large enough so just after the nuclei collide the temperature is that of the unverse about $10^{-5}$ seconds after the Big Bang, when the universe was too hot for protons or neutrons and consisted of quarks and gluons (the constituents of proton and nucleons)-the QGP.

As Figure 17 illustrates for Au-Au collisions, the emission of mixed hybrid mesons, the $\Psi(2 S)$ and $Y(3 S)$ as discussed above, with active gluons, could be a signal of the formation of the QGP.

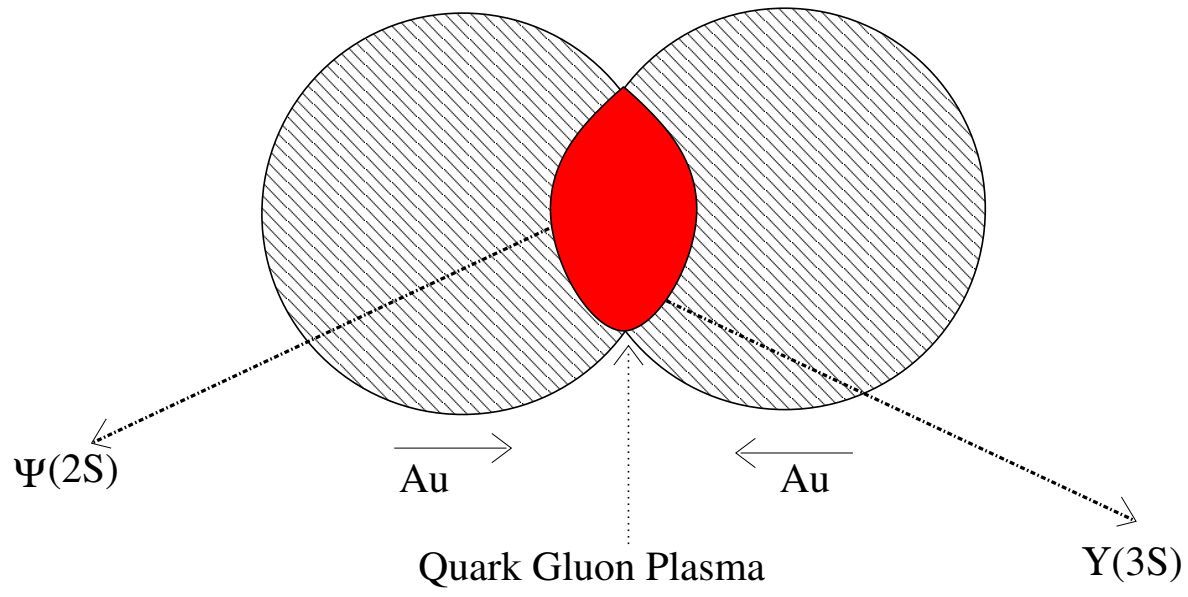

Figure 17. Au-Au collisions producing $\Psi(2 S)$ and $Y(3 S)$ from the QGP.

As discussed above the detection of the heavy quark mixed hybrid mesons $\Psi(2 S)$ and $Y(3 S)$ produced in Au-Au collisions is an experimental challenge. 


\section{Conclusions}

After a brief review of Quantum Chromodynamics QCD, Sum Rules were used to show that the $\Psi(2 S), \mathrm{Y}(3 S)$ are mixed hybrid states [11].

The conclusion from QCD sum rules that the $\Psi(2 S)$ and $Y(3 S)$ meson states are approxumastely half normal and half hybrid states was confirmed by the ratios of cross sections of $\Psi(2 S)$ to $J / \Psi(1 S)$ for $\mathrm{Cu}-\mathrm{Cu}$ Collisions and $\mathrm{Y}(3 S)$ to $\mathrm{Y}(1 S)$ for $\mathrm{p}$-p collisions compared to experimental results.

In our next section, the production of $\Psi(m S)$ and $Y(n S)$, with $m=1,2$ and $n=1,2,3$, in Cu-Cu and Au-Au collisions with $\sqrt{s_{p p}}=200 \mathrm{GeV}$ was reviewed, with experimental tests expected in the future. In our final section the creation of the QGP via A-A collisions and possible detection of the QGP via the emission of mixed hybrid mesons was reviewed. Since $\Psi(2 S)$ and $Y(3 S)$ are mixed hybrid states their production and detection in A-A collisions could provide evidence for the creation of the QGP.

Acknowledgments: L.S.K. acknowledges support in part as a visitor at the Los Alamos National Laboratory.

Funding: This research received no external funding.

Conflicts of Interest: The authors declare no conflict of interest.

\section{References}

1. Tanabashi, M. Particle Data Group. Phys. Rev. D 2018, 98, 030001. [CrossRef]

2. Cheng, P.-T.; Li, L.-F. Gauge Theory of Elementary Particle Physics, 1st ed.; Oxford University Press: Oxford, UK, 1988.

3. Kronfeld, A.S. Twenty-first century lattice gauge theory: Results from the quantum chromodynamics lagrangian. Annu. Rev. Nucl. Part. Sci. 2012, 62, 265-284. [CrossRef]

4. Shifman, M.A.; Vainstein, A.I.; Zakharov, V.I. QCD and resonance physics. theoretical foundations. Nucl. Phys. B 1979, 147, 385-447. [CrossRef]

5. Brambilla, N.; Eidelman, S.; Heltsley, B.K.; Vogt, R.; Bodwin, G.T.; Eichten, E.; Frawley, A.D.; Meyer, A.B.; Mitchell, R.E.; Papadimitriou, V.; et al. Heavy quarkonium: progress, puzzles, and opportunities. Eur. Phys. J. C 2011, 71, 1534. [CrossRef]

6. Rothkopf, A. Heavy Quarkonium in Extreme Conditions. arXiv 2019, arXiv:1912.02253.

7. Kisslinger, L.S.; Liu, M.X.; McGaughey, P. Heavy-quark-state production in $p-p$ collisions. Phys. Rev. D 2011, $84,114020$.

8. Bodwin, G.T.; Bratten, E.; Lepage, G.P. Rigorous QCD analysis of inclusive annihilation and production of heavy quarkonium. Phys. Rev. D 1995, 51, 1125. [CrossRef] [PubMed]

9. Cho, P.L.; Leibovich, A.K. Color-octet quarkonia production. Phys. Rev. D 1996, 53, 150. [CrossRef] [PubMed]

10. Braaten, E.; Chen, Y.-Q. Helicity decomposition for inclusive $J / \Psi$ production. Phys. Rev. D 1996, 54, 3216. [CrossRef] [PubMed]

11. Leonard, S.K. Mixed heavy quark hybrid mesons, decay puzzles, and RHIC. Phys. Rev. D 2009, 79, 114026.

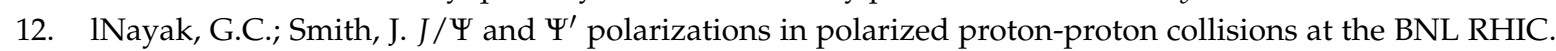
Phys. Rev. D 2006, 73, 014007.

13. Rohini M.G.; Guarav, M.; Saurabh, D.R. Looking for BSM physics using top-quark polarization and decay-lepton kinematic asymmetries. Phys. Rev. D 2015, 92, 094013.

14. Kisslinger, L.S.; Liu, M.X.; McGaughey, P. Heavy-quark-state production in A-A collisions at $\sqrt{s_{p p}}=200 \mathrm{GeV}$. Phys. Rev. C 2014, 89, 024914. [CrossRef]

15. Adare, A.; Afanasiev, S.; Aidala, C.; Ajitanand, N.N.; Akiba, Y.; Al-Bataineh, H.; Alexander, J.; Angerami, A.; Aoki, K.; Apadula, N. et al. Ground and excited state charmonium production in $p+p$ collisions at $\sqrt{s}=$ $200 \mathrm{GeV}$. Phys. Rev. D 2012, 85, 092004. [CrossRef]

16. Moreno, G.; Brown, C.N.; Cooper, W.E.; Finley, D.; Hsiung, Y.B.; Jonckheere, A.M.; Jostlein, H.; Kaplan, D.M.; Lederman, L.M.; Hemmi, Y.; et al. Dimuon production in proton-copper collisions at $\sqrt{s}=38.8 \mathrm{GeV}$. Phys. Rev. D 1991, 43, 2815. [CrossRef] [PubMed]

17. Liu, Y.; Greiner, C.; Kostyuk, A. $B_{c}$ meson enhancement and the momentum dependence in $\mathrm{Pb}+\mathrm{Pb}$ collisions at energies available at the CERN Large Hadron Collider. Phys. Rev. C 2009, 80, 041902. 
18. Adare, A. et al. [PHENIX Collaboration]. Energy loss and flow of heavy quarks in Au + Au collisions at $\sqrt{s_{N N}}=200 \mathrm{GeV}$. Phys. Rev. Lett. 2007, 98, 172301. [CrossRef]

19. Qiu,J.; Vitev,I. Resummed QCD Power Corrections to Nuclear Shadowing. Phys. Rev. Lett. 2004, 93, 262301.

20. Enqvist, K.; Ignatius, J.; Kajantie, K.; Rummukainen, K. Nucleation and bubble growth in a first-order cosmological electroweak phase transition. Phys. Rev. D 1992, 45, 3415. [CrossRef]

(C) 2020 by the authors. Licensee MDPI, Basel, Switzerland. This article is an open access article distributed under the terms and conditions of the Creative Commons Attribution (CC BY) license (http:/ / creativecommons.org/licenses/by/4.0/). 